\title{
Basic Research on 3D Cultural Heritage Packaging Technology Using Thermoplastic Polyurethane Elastomers
}

\author{
Seung-Jun $\mathrm{Oh}^{1}$, Koang-Chul $\mathrm{Wi}^{2},{ }^{*}$ \\ ${ }^{1}$ The Research Center of Conservation Science for Cultural Heritage, Hanseo University, Seosan 31962, Korea \\ ${ }^{2}$ Department of Cultural Heritage Conservation, Hanseo University, Seosan 31962, Korea
}

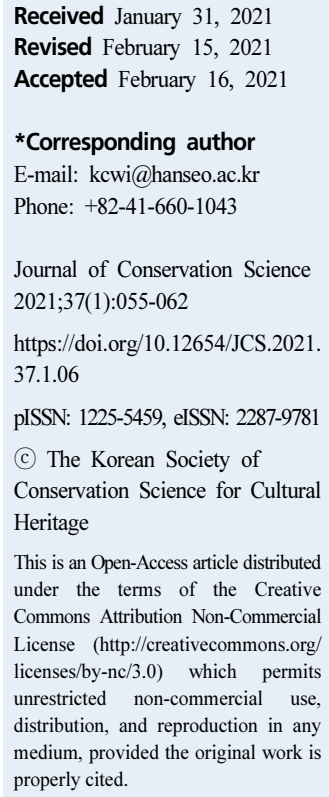

ABSTRACT This study investigated mechanical property changes by measuring compression factors, resilience, and compressive strength according to packaging pattern and filling rate to identify the applicability of cultural heritage packaging using thermoplastic polyurethane elastomers (TPU). Research results indicate that the cross-shaped $3 \mathrm{D}$ pattern showed the best resilience when the internal filling rate was $20 \%$, while the octet pattern was the best when the filling rate was either 40 and $60 \%$. The octet pattern had the best mechanical properties and stability with resistance capacities of $20.79 \mathrm{kgf} / \mathrm{cm}^{2}$, $40.40 \mathrm{kgf} / \mathrm{cm}^{2}$, and $82.23 \mathrm{kgf} / \mathrm{cm}^{2}$ at $38 \%, 39 \%$, and $40 \%$ recovery speeds, respectively, depending on the internal filling rate $(20,40,60 \%)$. Based on these results, basic data on the applicability, stability, and reliability of $3 \mathrm{D}$ cultural heritage packaging materials using TPU materials were obtained.

Key Words Thermoplastic polyurethane elastomers (TPU), Cultural heritage packaging, Resilience, Recovery speed, Resistance capacity packaging material, Conservation science

\section{INTRODUCTION}

Packaging refers to a technology or technique that using a box, container, or foaming agent, protects the value and present condition of a specific product from being damaged, providing convenience and stability in transporting or storing products. Materials used for packaging are generally glass, plastic, paper, etc., and a filler such as a foaming agent or air cap is used to secure the product's safety through shock absorption. Such packaging is often used in various fields, including the food, stationery, and clothing industries. Moreover, packaging is an essential means to safely store cultural heritage items such as relics, cultural properties, artworks, and crafts and to prevent damage during transportation for exhibition purposes.

The packaging of cultural heritage items stored in museums, art galleries, and cultural property research institutions is necessary to protect from physical shocks that may occur when transported to a specific place for exhibitions and other environments, and from the existing storage settings (Shin, 2001). Surface packaging materials such as neutral paper, neutral hanji, tie bags, cotton wrapping, foaming agents, filling agents such as DuPont and sponges, foam board boxes, corrugated boxes, inner skin boxes such as paulownia boxes, and outer skins such as wooden boxes and aluminum boxes are currently used (Lee, 2018). These packaging materials are applied differently depending on the size and shape of the cultural heritage to be displayed, the 
current state, the distance traveled, and the environment, used to consider stability, economy, workability, and usability based on buffering and resilience (Shin, 2018).

However, despite their various advantages, surface packaging materials have weak resilience and cushioning power; cotton bags, for example, have rough surfaces among cushioning materials (Kim, 2018) and generates a large amount of dust. Foaming agents and DuPont have the disadvantage of relatively weak buffering power and resilience. Sponges, despite their high cushioning and resilience, have low economic value and workability. Inner and outer boxes take a long time to manufacture, have low economic efficiency, and are difficult to reuse (Jeon, 2018). The use of packaging materials differs depending on the curator, conservation scientist, or packaging specialist; moreover, packaging technology is not standardized according to packaging materials, packaging techniques, and packaging objects. Since the 1980s, although changes have been implemented based on packaging techniques, research and development of packaging technologies at a large scale remain insufficient.

This study attempts to identify the applicability of cultural heritage packaging technology using 3D printouts using filaments made of thermoplastic polyurethane elastomers (TPU). TPU materials can polymerize various hardness elastomers according to the ratio of hard and soft phases through block copolymerization. It has excellent chemical resistance, including oil resistance and fuel resistance, and optimal mechanical properties, such as tensile strength, tear strength, and flexibility due to its combination with urethane (Hong, 2015).

As their characteristics reduce the weight of the packaging material and prevent dust generation and damage to artifacts due to rough surfaces, TPU have been selected as cultural heritage packaging materials owing to their shock absorption and resilience due to their high elasticity, low strain rate at low temperature, and stable physical properties. In addition, 3D packaging techniques using TPU filaments have been used to standardize packaging technologies according to the shape, size, material, environment, etc., of the cultural heritage item. Moreover, packing personnel and researchers have carried out a basic study to apply this packaging technology to reuse packaging materials.

\section{MATERIALS AND METHODS}

\subsection{D specimen}

A specimen was printed with a stacked-type 3D printer (B420, STELLAMOVE, KOR) using a TPU filament. For the output's internal filling pattern, three types of quarter-cubic, cross-shaped 3D, and 3D-structure octet were selected (Figure 1); this is because the $\mathrm{X}$-axis and $\mathrm{Y}$-axis filling

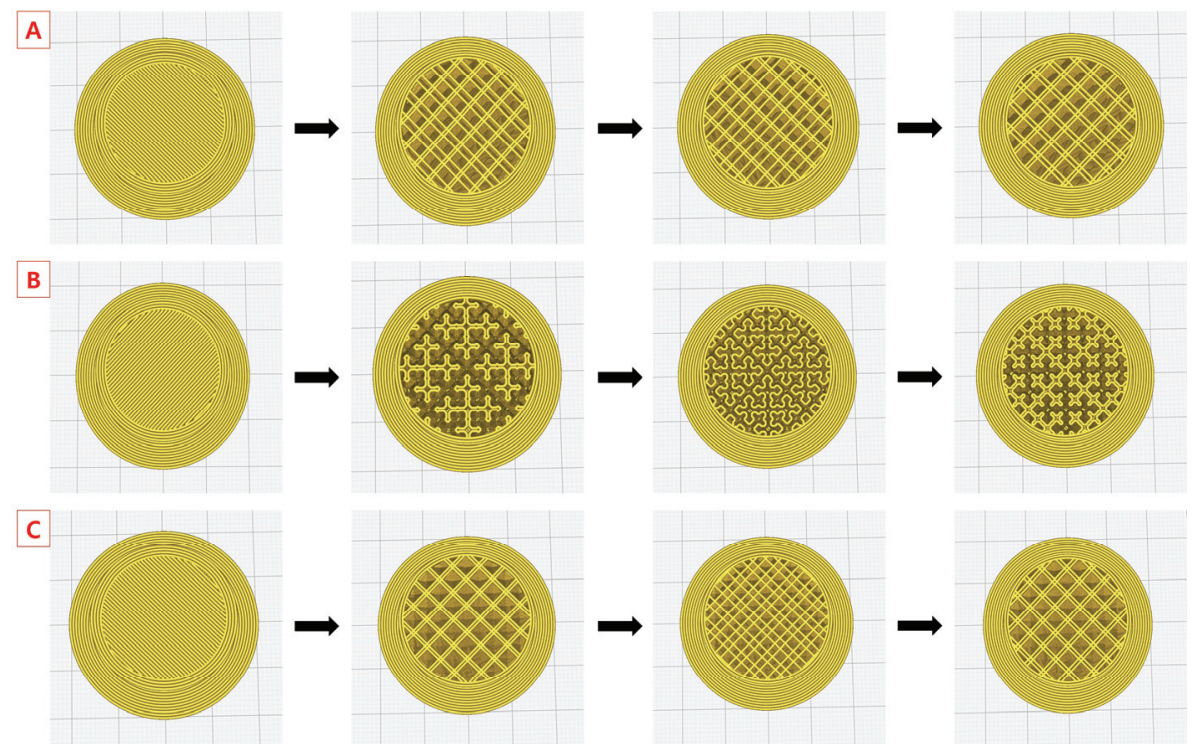

Figure 1. Inner filling pattern by pattern; (A) Quarter cubic, (B) Cross-shaped 3D, (C) Octet. 
patterns are output differently after printing, even within the same pattern, owing to the characteristics of the stacking method. This was considered as testing under such conditions was impossible. The printing condition was applied equally to three outputs (line width of $0.6 \mathrm{~mm}$, wall thickness of 1.2 $\mathrm{mm}$, lower and upper thickness of $1.2 \mathrm{~mm}$ ); the closer the filling density was to $100 \%$, the higher the internal filling density. As the density was too high, the shock absorption, elasticity, and resilience were significantly lowered, and each pattern was output at $20 \%, 40 \%$, and $60 \%$.

\subsection{Physical property test}

The compression factor, rebound elasticity, and compressive strength tests were conducted as physical property tests. However, as there is no standard specification for 3D-printed packaging using TPU materials, a physical property test was performed on a test item using an elastic packaging material impact absorbing pad. A compression factor test (KS M 6518: physical test method for vulcanized rubber) measured the compression factor due to the heat compression of vulcanized rubber used in a part subjected to static compression or shearing force (Jin et al., 2009). The specimen had a cylindrical shape with $12.70 \pm 0.050 \mathrm{~mm}$ and $29.00 \mathrm{~mm}$ (Figure 2). The prepared specimen was compressed for $22 \mathrm{~h}$ with a compression device sufficiently preheated to the specified temperature (70 \pm $1{ }^{\circ} \mathrm{C}$ ), and the compression factor was measured (Joubert, C et al., 2003).

The rebound elasticity test (KS M 6518: physical test method for vulcanized rubber) is a method of checking the rebound height value by dropping an iron bar freely and measuring the rebound elasticity of urethane and rubber materials using a rebound resilience test device. The specimen was made in a cylindrical shape with a thickness of $12.70 \pm 0.050 \mathrm{~mm}$ and diameter of approximately 29.00 $\mathrm{mm}$ (Figure 3). The compression strength test was performed using a universal testing machine (AG-X plus $5 \mathrm{kN}$, Shimadzu, JPN), according to KS ISO3386-1 (soft foamed polymer material).
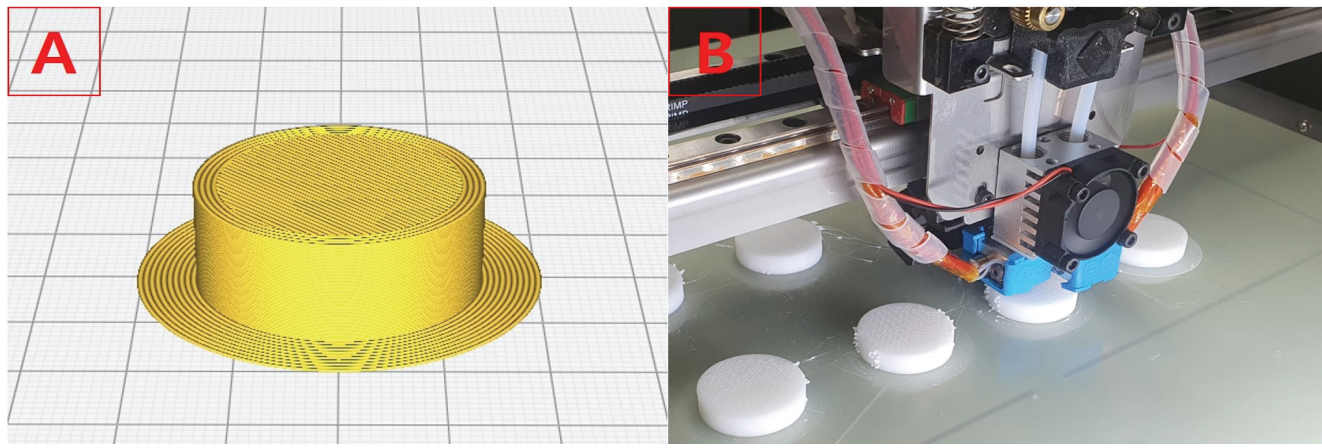

Figure 2. Specimen output; (A) specimen modeling image, (B) specimen output process.

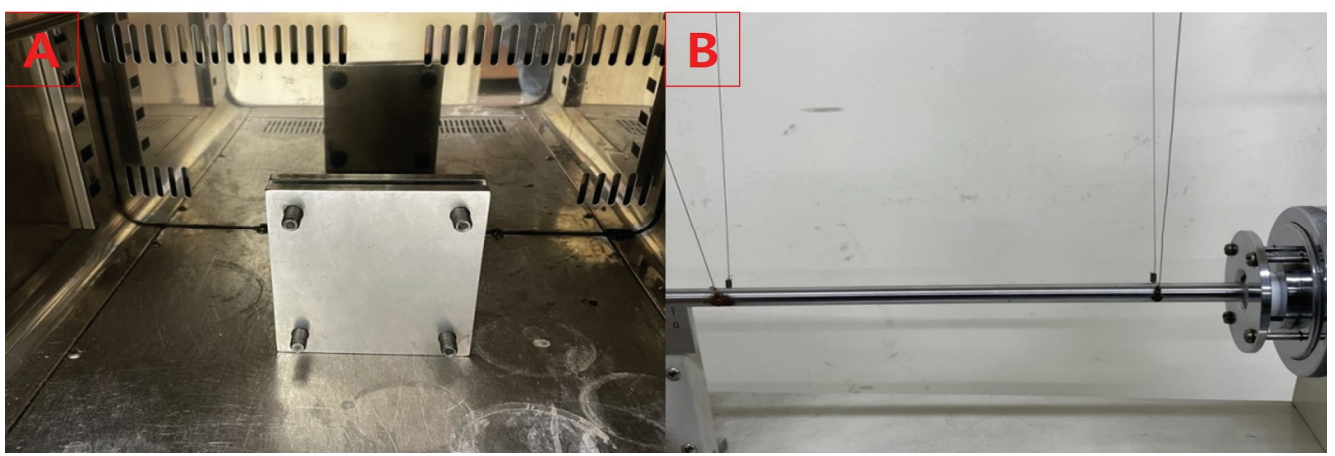

Figure 3. Physical Property Test; (A) Compression factor, (B) Resilience according. 


\section{RESULTS AND CONSIDERATIONS}

\subsection{D specimen}

The 3D specimen was printed using a TPU filament in a stacked manner. Three types of internal filling patterns, namely, quarter-cubic, cross-shaped 3D, and octet 3D structures, were used. The overlapping ratio between the inner filling pattern line and outer wall was set as $15 \%$, the upper and lower patterns were printed in a line form, and the printing and filling speeds were set to $15 \mathrm{~mm} / \mathrm{s}$ considering the TPU filament's characteristics according to the specimen's suitability for each physical property test (Figure 4).

\subsection{Physical property test}

The compression factor according to the internal filling rate is shown in Table 1 and is expressed by the following equation (Jin et al., 2011):

$$
\begin{array}{ll} 
& \text { C: Compression set }(\%) \\
C=\frac{t_{0}-t_{1}}{t_{0}-t_{2}} \times 100 & \begin{array}{l}
\mathrm{t}_{0}: \text { Original thickness of specimen } \\
(\mathrm{mm})
\end{array} \\
& \mathrm{t}_{1}: \text { Final thickness of specimen }(\mathrm{mm}) \\
& \mathrm{t}_{2}: \text { Thickness of space bar: } 9.52 \mathrm{~mm}
\end{array}
$$

A $74 \%$ compression factor was found in the quarter-cubic pattern at a $20 \%$ internal filling; $71 \%$ and $72 \%$ compression factors were observed at $40 \%$ and $60 \%$ internal fillings, respectively. This is because the opposite of the compression

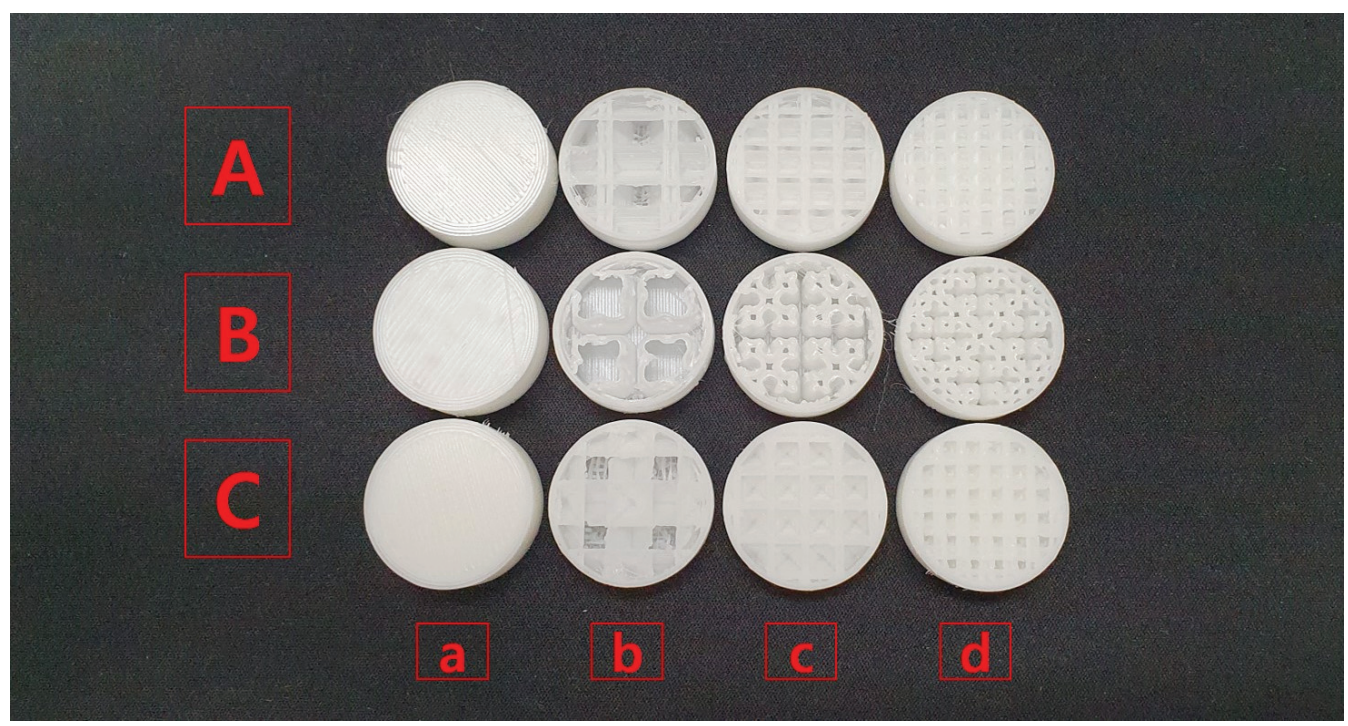

Figure 4. TPU 3D output specimen; (A) Quarter Cubic, A-a: final specimen, A-b: inside specimen with internal filling rate $20 \%$, A-c: inside specimen with internal filling rate $40 \%$, A-d: inside specimen with internal filling rate $60 \%$, (B) Cross shape 3D, B-a: final specimen, B-b: inside specimen with internal filling rate $20 \%$, B-c: inside specimen with internal filling rate $40 \%$, B-d: inside specimen with internal filling rate $60 \%$, (C) The octet, C-a: final specimen, C-b: Inside the specimen with an internal filling rate of $20 \%$, C-c: Inside the specimen with an internal filling rate of $40 \%$, C-d: Inside the specimen with an internal filling rate of $60 \%$.

Table 1. Compression factor measurement result (\%)

\begin{tabular}{cccc}
\hline Physical property & Quarter cubic & Cross shaped 3D & Octet \\
\hline $20 \%$ & 74 & 60 & 70 \\
\hline $40 \%$ & 71 & 80 & 70 \\
\hline $60 \%$ & 72 & 74 & 69 \\
\hline
\end{tabular}


factor measured in the TPU subjected to heating static compression indicates either circular resilience or regular resilience, resulting in a lower value the higher the resilience. The specimen produced with quarter-cubic pattern had the highest resilience when the internal filling rate was $40 \%$. The specimen printed as a cross-shaped 3D pattern showed compression factors of $60 \%, 80 \%$, and $74 \%$ when the internal filling rates were $20 \%, 40 \%$, and $60 \%$, respectively. Contrary to the quarter-cubic pattern, the highest compression factor was observed when the internal filling rate was $40 \%$, and the highest resilience was observed at an internal filling rate of $20 \%$. In the octet filling pattern, the same compression factor (70\%) was observed at $20 \%$ and $40 \%$ filling rates; when the filling rate was $60 \%$, a slight change with a compression factor of $69 \%$ was observed.

At the same internal filling rate $(20 \%)$, the quarter-cubic pattern showed the highest compression factor, while the lowest compression factor was found in the cross-shaped 3D pattern. On the other hand, at an internal filling rate of $40 \%$, the cross-shaped 3D pattern showed the lowest resilience, with the highest compression factor of $80 \%$. An internal filling rate of $60 \%$ was found in the order of cross-shaped 3D, quarter-cubic, and octet patterns (Figure 4). These test results indicated that the packaging material printed using the octet pattern minimizes the change in resilience depending on the internal filling rate. The packaging materials printed in the quarter-cubic and cross-shaped 3D patterns have different resilience values depending on the size, weight, and shape of the cultural heritage item (Figure 5).

In the resilience test, it is possible to check the recovery speed when the packaging material is deformed by external impacts or loads different from the compression factor's stability and recovery degree. The measurement results are presented in Table 2. The specimens printed with the quarter-cubic pattern showed a $35 \%$ resilience modulus when the internal filling rate was $20 \%$, while $40 \%$ and $39 \%$ resilience moduli were identified at filling rates of $40 \%$ and $60 \%$, respectively. Therefore, if the packaging material with the quarter-cubic pattern is used, the internal filling rate is set to $40 \%$ or more, and a higher recovery rate can be obtained from impact. When applying the cross-shaped 3D pattern, the same resilience modulus of $32 \%$ was identified when the internal filling rates were $20 \%$ and $40 \%$. Simultaneously, a resilience modulus of $39 \%$ was observed at a filling rate of $60 \%$. Therefore, it is interpreted that the recovery speed is not proportional to the internal filling rate of the pattern but depends on the pattern shape.

In the octet pattern, when the internal filling rates were $20 \%, 40 \%$, and $60 \%$, the resilience moduli were measured to be $38 \%, 39 \%$, and $40 \%$, respectively, indicating that the increases in the filling rate and resilience modulus were proportional. The resilience modulus pattern that constantly
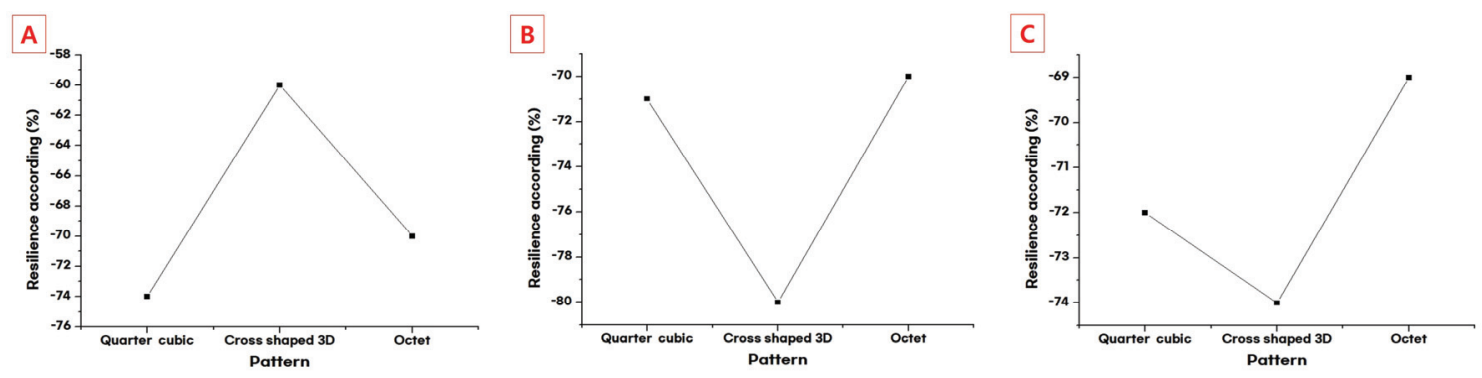

Figure 5. Resilience according to the filling pattern and ratio; (A) Resilience at 20\% internal filling rate (\%), (B) Resilience at $40 \%$ internal filling rate (\%), (C) Resilience at $60 \%$ internal filling rate $(\%)$.

Table 2. Resilience elasticity measurement result (\%)

\begin{tabular}{cccc}
\hline Physical property & Quarter cubic & Cross shaped 3D & Octet \\
\hline $20 \%$ & 35 & 32 & 38 \\
\hline $40 \%$ & 40 & 32 & 39 \\
\hline $60 \%$ & 39 & 39 & 40 \\
\hline
\end{tabular}


rises as described above allows the application range to be systematically set when selecting a packaging material suitable for items with cultural heritage characteristics. If a more detailed filling rate test and shock absorption measurement are performed, patterns that are not proportional to the internal filling rate can be used as a stable packaging material by acting simultaneously with resilience and shock absorption for each pattern (Figure 6).

The compressive strength measurement tests the compressive strength and resistance capacity generated during transport, packing, and loading by checking the degree to which the packing structure withstands external pressures and loads; the results are listed in Table 3. The quarter-cubic pattern was found to be $15.83 \mathrm{kgf} / \mathrm{cm}^{2}$ at an internal filling rate of $20 \%, 40.15 \mathrm{kgf} / \mathrm{cm}^{2}$ at $40 \%$, and $54.17 \mathrm{kgf} / \mathrm{cm}^{2}$ at $60 \%$. As the filling rate increased, the resistance capacity increased. The cross-shaped 3D pattern was $13.47 \mathrm{kgf} / \mathrm{cm}^{2}$ at a filling rate of $20 \%, 37.56 \mathrm{kgf} / \mathrm{cm}^{2}$ at $40 \%$, and 66.76 $\mathrm{kgf} / \mathrm{cm}^{2}$ at $60 \%$. The octet pattern yield $20.79 \mathrm{kgf} / \mathrm{cm}^{2}, 40.40$ $\mathrm{kgf} / \mathrm{cm}^{2}$, and $82.23 \mathrm{kgf} / \mathrm{cm}^{2}$ at fillings rates of $20 \%, 40 \%$, and $60 \%$, respectively. In all the patterns, as the internal filling rate increased, the compressive strength increased.

Figure 7 shows the changes in compressive strength according to the internal filling rate using quarter-cubic, cross-shaped 3D, and octet patterns. As the rapid decreases according to the resilience of the TPU material's characteristics was challenging to visualize through a graph, the first transition point result in the rising curve was presented. The cross-shaped 3D pattern distinguishes between the rising and falling curves and has a pattern structure different from the quarter-cubic and octet patterns. This is considered a changing aspect resulting from the filling rate
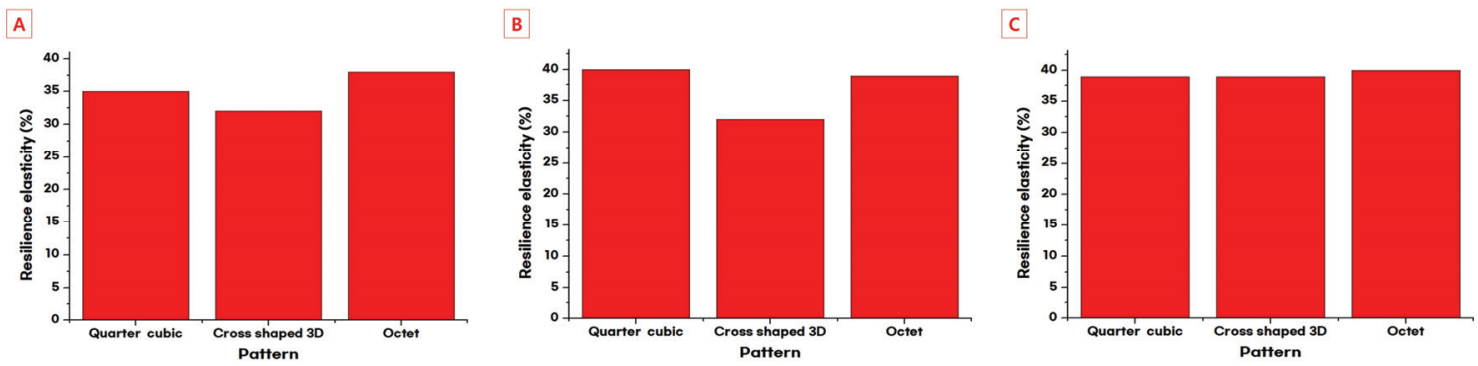

Figure 6. Resilience elasticity to the filling pattern and ratio; (A) Resilience modulus (\%) at $20 \%$ internal filling rate, (B) Resilience modulus (\%) at 40\% internal filling rate, (C) Resilience modulus (\%) at 60\% internal filling rate.

Table 3. Compressive Strength measurement result $\left(\mathrm{kgf} / \mathrm{cm}^{2}\right)$

\begin{tabular}{cccc}
\hline Intemal filling rate & Quarter cubic & Cross shaped 3D & Octet \\
\hline $20 \%$ & 15.83 & 13.47 & 20.79 \\
\hline $40 \%$ & 40.15 & 37.56 & 40.40 \\
\hline $60 \%$ & 54.17 & 66.76 & 82.23 \\
\hline
\end{tabular}
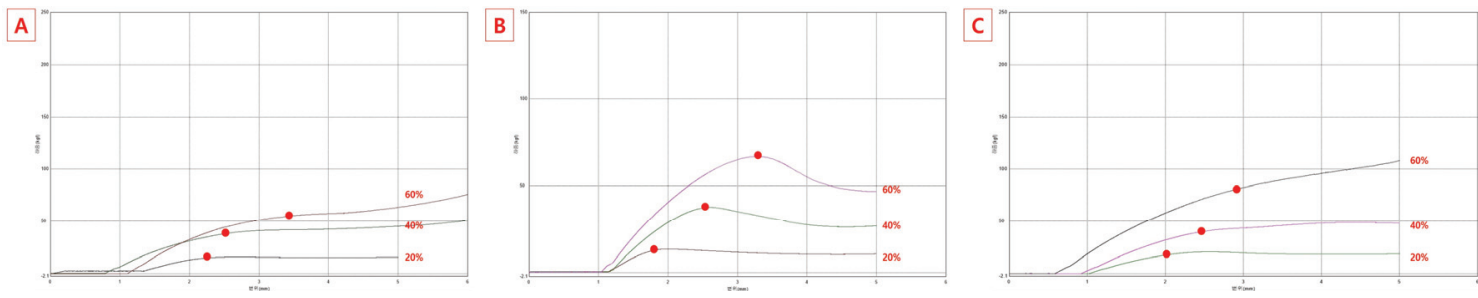

Figure 7. Resistance capacity according to the filling pattern and ratio; (A) Resistance capacity according to the internal filling rate of the quarter cubic $\left(\mathrm{kgf} / \mathrm{cm}^{2}\right)$, (B) Resistance capacity according to the internal filling rate of the cross-shaped $3 \mathrm{D}\left(\mathrm{kgf} / \mathrm{cm}^{2}\right)$, (C) Resistance capacity according to the internal filling rate of the octet $\left(\mathrm{kgf} / \mathrm{cm}^{2}\right)$. 
according to the pattern direction and shape. Quarter-cubic and octet patterns with a similar structure show similar rising curves. However, when the internal filling rate was $60 \%$ in octet patterns, they showed a continuous rising curve, believed to be due to the internal filling rate and direction of the pattern. In addition, showing a continuous rising curve compared to the cross-shaped 3D pattern is likely because resistance capacity appears different depending on the pattern shape and the internal filling rate rather than the TPU material's elasticity. This means that the utilization and application range can be set in various ways as it is possible to provide custom production according to the item to be packed and the external environment.

\section{CONCLUSION}

In this study, to check the applicability of cultural heritage packaging materials using TPU filaments, a comparative experiment on the change in mechanical properties was conducted by measuring the compression factor, resilience elasticity, and compressive strength according to the pattern and filling rate inside packaging material; the following results were obtained:

1) Resistance to external impact and pressure was checked to determine the compression factor. When the internal filling rate was $20 \%$, the circular resilience of the packaging material made of a cross-shaped 3D structure was the most optimal. In addition, at filling rates of $40 \%$ and $60 \%$, the octet pattern's resilience was the best. Therefore, it is believed that a packaging material to protect cultural heritage items from impact and pressure stronger than the cross-shaped 3D structure can be produced.

2) Resilience elasticity is the speed at which the packaging material recovers to its original shape when the packaging material is deformed due to external impact or load. When the packaging material is made of a quarter-cubic and octet pattern, the recovery rate is high. In the cross-shaped 3D pattern structure, the recovery rate was the lowest, except at a filling rate of $60 \%$. A stability proportional to the filling rate was also determined.

3) Compressive strength can show the resistance capacity of pressure and load during packaging, loading, and transportation. The highest resistance capacity was observed in the octet pattern. The quarter-cubic pattern showed a high resistance capacity at $20 \%$ and $40 \%$ filling rate, and the cross-shaped 3D pattern showed excellent resistance capacity at a filling rate of $60 \%$. As the optimal pattern and internal filling rate are determined when the resistance-capacitance is proportional, it is assumed that the thickness of the outer wall of the specimen and overlapping ratio of the pattern and outer wall are constant and output; therefore, further research is needed.

Based on these conclusions, to apply packaging materials for cultural heritage items using TPU, stability and reliability must be confirmed through various tests and applicability studies, such as fatigue, shock absorption, tear strength, and aging tests by repeated loads. This study presents basic data in this field of research with TPU materials.

\section{ACKNOWLEDGMENTS}

This research was supported by Basic Science Research Program through the National Research Foundation of Korea (NRF) funded by the Ministry of Education (2020R1I1A 1A01071331).

\section{REFERENCES}

Hong, S.G., 2015, Synthesis and Properties of the Thermoplastic Polyurethane containing Inoranic Filler. Master dissertation, Pusan National University, Busan, 1-7. (in Korean with English abstract)

Jeon, J.J., 2018, Collection packaging cases and research on packaging materials improvement: Examples of packaging methods and materials for American and European cultural properties. Gyeongju National Museum Symposium, Gyeongju, Dec 14, 3-8. (in Korean)

Jin, H.H., Hong, C.K., Cho, D.L. and Kaang, S.Y., 2009, Effects of temperature and curing systems on compression set of NR compounds at constant load. Elastomers and Composites, 44(1), 41-46. (in Korean with English abstract)

Jin, S.H., Hong, J.H., Kim, I., Yun, J.H. and Shim, S.E., 2011, Effect of vinyltriethoxysilane content on mechanical and physical properties of precipitated silica reinforced silicone rubber. Polymer (Korea), 35(4), 342-349. (in 
Korean with English abstract)

Joubert, C., Michel, A., Choplin, L. and Cassagnau, P., 2003, Influence of the crosslink network structure on stress-relaxation behavior: viscoelastic modeling of the compression set experiment. Journal of Polymer Physics, 41(15), 1779-1790.

Kim, S.K., 2018, Collection packaging cases and research on packaging materials improvement: A study on packaging of art in Asia. Gyeongju National Museum Symposium, Gyeongju, Dec 14, 29-35. (in Korean)

Lee, J.Y., 2018, Collection packaging cases and research on packaging materials improvement: Research on packaging materials for transport and storage of collections and improvement of packaging methods, Gyeongju National Museum Symposium, Gyeongju, Dec 14, 83-89. (in Korean)

Shin, M.K., 2001, Study on the packaging of the museum collections: focused on the temperature \& relative humidity test and vibration \& impact test, Master dissertation, Yong-In University, Yongin, 1-3. (in Korean with English abstract)

Shin, H.Y., 2018, Collection packaging cases and research on packaging materials improvement: Study on the packaging method of Korean cultural properties, Gyeongju National Museum Symposium, Gyeongju, Dec 14, 73-76. (in Korean) 